\title{
TTR
}

Traduction, terminologie, re?daction

\section{Comprendre, évaluer, prévenir} Pratique, enseignement et recherche face à l'erreur et à la faute en traduction

\section{Daniel Gouadec}

Volume 2, numéro 2, 2e semestre 1989

L'erreur en traduction

URI : https://id.erudit.org/iderudit/037045ar

DOI : https://doi.org/10.7202/037045ar

Aller au sommaire du numéro

Éditeur(s)

Association canadienne de traductologie

ISSN

0835-8443 (imprimé)

1708-2188 (numérique)

Découvrir la revue

Citer cet article

Gouadec, D. (1989). Comprendre, évaluer, prévenir : pratique, enseignement et recherche face à l'erreur et à la faute en traduction. TTR, 2(2), 35-54.

https://doi.org/10.7202/037045ar d'utilisation que vous pouvez consulter en ligne.

https://apropos.erudit.org/fr/usagers/politique-dutilisation/ 


\section{Comprendre, évaluer, prévenir}

\section{Pratique, enseignement et recherche face à l'erreur et à la faute en traduction}

\section{Daniel Gouadec}

Il n'est nulle pratique de la traduction, nul enseignement de traduction, nulle recherche fondamentale ou appliquée portant sur la traduction qui ne renvoie, implicitement ou explicitement, à la notion d'erreur. Rares sont cependant les systèmes d'évaluation pédagogique ou professionnelle aux critères objectivement définis. Plus rares encore sont les procédures d'enseignement et de formation prenant en compte les mécanismes fondamentaux des erreurs. Rarissimes sont les travaux et publications proposant une analyse des comportements générateurs d'erreurs de traduction, de leurs causes et de leurs effets. Et l'on serait sans aucun doute bien en peine de citer une définition de l'erreur en traduction qui soit autre chose qu'une pseudo-description de l'effet de la "faute».

La notion d'erreur en traduction étant omniprésente dans les arrière-pensées du traducteur (réviseur ?), du pédagogue (didacticien ?), et du chercheur (cogniticien?), pareille situation est pour le moins regrettable ... à moins que tout un chacun ne fasse référence au système absolu hérité des notations de versions et thèmes d'apprentissage linguistique et reposant sur des types ou catégories de "fautes " dont les appellations se rattachent à la série enchaînant le non-sens, le contresens, le faux sens, le barbarisme, et le reste. 
Le "système" d'évaluation ainsi installé présente de multiples avantages. Il est d'une très grande simplicité. Il propose des descriptions apparemment «transparentes » des fautes. Il permet d'affecter directement ou indirectement des coefficients de pénalité-sanction aux divers types d' " erreurs ». Issu du contexte pédagogique, il s'adapte au contexte professionnel moyennant quelques menus aménagements. Il accepte toute forme de corrélation avec le catalogue d'erreurs (erreurs-type ou erreurs atypiques) établi par le réviseur, l'enseignant, et parfois même le chercheur.

Mais tout repose sur une ambiguïté linguistique inhérente aux désignations : chaque catégorie du système standard et de ses divers avatars désigne avant tout un palier sur l'échelle des sanctions-pénalités applicables à l'effet (la faute) de l'erreur dont le mécanisme est ainsi occulté. Le système n'est simple que parce qu'il est rudimentaire; il court-circuite toute explication ; il est anti-pédagogique en ce sens qu'il ne «démonte" rien et qu'il repose exclusivement sur l'évaluation négative par cumul de pénalités ; il empêche que les chercheurs s'interrogent sur les stratégies de la traduction et sur les comportements des traducteurs. En un mot, il dispense de comprendre, analyser, évaluer, corriger, prévenir, les comportements induisant l'erreur, les incidences du risque d'erreur sur les comportements du traducteur et, bien entendu, l'incidence de l'erreur sur la qualité de la traduction.

La pérennité du système dit des "catégories universelles de fautes» s'explique par la multiplicité des points de convergence avec les grands courants idéologiques et avec les habitudes. Tout est en effet parfait si la traduction correspond au transfert d'unités linguistiques (lexicales et syntaxiques) minimales constituant autant de supports potentiels de fautes ou «pièges » dont les catégories sont répertoriables et justiciables d'un traitement d'écart-type (par "procédés de traductions »). On comprend pourquoi la pratique pédagogique et professionnelle se réduit généralement, après proclamation de grands principes, au traitement de petits points pour prévenir des fautes d'autant plus "grosses " que, ayant fait l'objet d'une mise en garde et d'un apprentissage, elles sont devenues « impardonnables".

Caricature? Oui. Certes. Caricature qu'une activité qui, se prétendant industrielle (à moins que son classement au nombre des industries de la langue ne soit une imposture), continue de se dispenser de procédures de contrôle de qualité. Caricature aussi qu'une recherche qui, se prétendant scientifique (à moins qu'il ne s'agisse d'un abus de langage), doive renoncer à l'expérimentation, à la mesure, et à l'analyse des processus et notamment des processus induisant l'erreur.

\section{Les postulats}

Quitte à leur trouver grâce au bout du compte, il faut, pour éviter les pièges idéologiques, remettre en question tous les principes courants 
de l'évaluation et même la terminologie en vigueur. Il importe surtout de formuler clairement les postulats sur lesquels se fonde l'analyse et qui sont au nombre de dix.

1. L'erreur s'inscrit dans le schéma:
a) existence d'un facteur de risque ;
b) existence d'un facteur aggravant le risque ;
c) déclenchement du mécanisme de l'erreur;
d) nature de l'erreur effective ;
e) incidence ou impact de l'erreur effective ;
f) traitement de la "faute " en contrôle de qualité.

2. Pédagogie, pratique, et recherche doivent s'interdire de générer des catégories rigides dont les valeurs seraient absolues.

3. L'erreur est à l'origine de la faute qui est elle-même à l'origine de l'éventuelle pénalité.

4. Chaque erreur est toujours, de par ses supports et son incidence, un cas d'espèce.

5. Les catégories d'erreurs ne peuvent se fonder que sur des paramètres de nature et d'impact sur lesquels se greffent, en amont, les causes d'erreur et, en aval, les pénalités pour «faute».

6. L'erreur résulte toujours d'un non-choix ou d'un mauvais choix qui peut être mauvais choix de l'opérande (élément sur lequel porte l'opération) ou mauvais choix de l'opération au moment considéré.

7. Les mécanismes de contrôle ou de prévention de la propagation des éventuels cônes de distorsion doivent être compris, analysés et, au besoin, corrigés.

8. Les erreurs de stratégie sont invariablement chaînées en ce sens que toute erreur initiale en entraîne d'autres. Il importe donc de construire des paliers homogènes au niveau desquels seront définis des objectifs cohérents de prévention d'erreurs.

9. Toute erreur n'est pas nécessairement négative.

10. Puisqu'il n'est de référence à l'erreur qu'avec arrière-pensées d'évaluation, il importe également d'affirmer clairement que :

a) la correction d'éventuelles erreurs (révision) ne peut ni ne doit se confondre avec l'évaluation de la qualité d'une traduction (impliquant une inévitable "mesure " de l'impact des distorsions engendrées par ces éventuelles erreurs). II faut donc éviter de confier à une même personne les fonctions de correcteur-réviseur et d'évaluateur d'une même traduction à moins que le système d'évaluation ne neutralise pleinement l'effet des éventuels «emportements" du révi- 
seur. Ceci demeure vrai lorsque l'évaluation prend en compte l'indice de révision nécessaire.

b) l'évaluation de la traduction ne peut en aucun cas valoir évaluation du traducteur;

c) l'évaluation ne peut se construire exclusivement sur une démarche négative de calcul de pénalités;

d) l'évaluation pédagogique ne se justifie qu'à la condition d'avoir des effets didactiques;

e) sauf identité de conditions, l'évaluation professionnelle n'est «juste» que si elle s'appuie sur une échelle relative prenant en compte la diversité des cas d'espèce que sont les textes.

\section{Nature et caractères de l'erreur}

La notion étant fortement polysémique, l'erreur en traduction n'est pas aisément définie. Nous choisirons donc d'aller progressivement d'une définition générique à une liste de caractères fonctionnels.

L'erreur en traduction est, d'un point de vue générique, une rupture de congruence dans le passage d'un document premier (à traduire, existant, compris, analysé) à un document second (à venir). En d'autres termes, l'erreur est distorsion injustifiée d'un message et/ou de ses caractères. Cette distorsion se juge par rapport à :

a) l'ensemble des règles génériques de communication (distorsion par effet absolu de communication)

et

b) l'ensemble des déterminants d'un projet de traduction (distorsion par effet relatif de transfert).

L'erreur absolue est indépendante de tout effet de traduction. Elle correspond à toute transgression injustifiée des règles de la grammaire culturelle (logique, formation des concepts, organisation et interprétation des concepts et de leurs interrelations), des règles de la grammaire linguistique (syntaxe, orthographe, etc.), ou des règles d'usage fondant les stéréotypies génériques ou spécifiques (type de texte, phrasologie, terminologie, etc.).

L'erreur relative est induite par a) la non-formation ou b) la non-formation adéquate ou c) le non-respect, d'un ou plusieurs des déterminants du projet de traduction agissant aux niveaux successifs du texte, du chapitre, de la section, de la rubrique, de la phrase, de la proposition (de tout segment considéré comme unité de transfert ou unité d'évaluation). Ces déterminants sont:

a) des déterminants externes:

* cadres de domaine et/ou secteurs de référence

* cadre de chronologie 
* cadre géographique

* public destinataire

* exploitation(s) prévue(s) - finalités

b) des déterminants internes

$*$ objet(s)

* thème(s) générique(s)

* trajet générique (incluant le type)

Entre les deux groupes peut intervenir un ensemble de déterminants internes-externes correspondant, par exemple, à la nature des découpages et aux modes de présentation de la traduction (alinéas, enrichissement de caractères, pagination, etc.)

Pour que les choses soient claires, il faut insister très fortement sur le fait que la distorsion par effet de traduction s'apprécie toujours par rapport aux conditions spécifiques de la traduction concernée et, singulièrement, de la nature du projet de traduction. Il faudrait donc

a) que toute évaluation soit fondée sur la définition d'un cahier des charges très précis et/ou le jugement d'échantillons préalablement à la traduction définitive,

b) que l'erreur soit toujours traitée de manière prospective et que toutes les décisions imposées par la conjonction d'un texte premier, d'un projet de traduction, et d'écarts culturels - linguistiques - rhétoriques aient effectivement été prises avant que ne débute la fabrication-rédaction du document second,

c) que l'analyse des facteurs de risque et des facteurs aggravants prenne en compte la variabilité des conditions de traduction.

Mais l'élément capital reste le niveau auquel se juge l'erreur. Les déterminants du projet et du trajet de traduction sont emboîtés par niveaux en ce sens que le projet intéressant la totalité du texte enchâsse les projets correspondant aux chapitres qui, à leur tour, enchâssent les projets correspondant aux sections, et ainsi de suite. Inévitablement, les critères et degrés de convergence/congruence ou, inversement, de distorsion, s'apprécient différemment selon le niveau ultime auquel s'arrêtent les emboitements de projets-trajets de traduction-transfert : si la congruence se définit au niveau du texte, la traduction est de type global, post-synthétique, absolue ( « tout » traduit) ou synoptique ; si l'unité de référence est le constituant le plus étroit, la réalisation et l'évaluation de la traduction portent nécessairement sur des unités elles-mêmes étrécies et cumulatives.

Sauf stipulation ${ }^{1}$ de l'unité minimale sur laquelle porte le projet de transfert, on retrouve le modèle implicite de la traduction absolue

1. La stipulation peut être directe (par intégration de directives explicites au cahier des charges) ou indirecte (par jugement sur échantillons). 
dans laquelle l'erreur s'évalue par référence aux seuls écarts-types entre langues-cultures dans une perspective macro-contextuelle de recouvrement de types de textes ou documents. On retrouve aussi le schéma de la pédagogie traditionnelle visant à répertorier des modalités de mise en place de ponts linguistiques entre unités syntaxiques réputées équivalentes par delà les écarts-types et sanctionnant des fautes-types qui seraient invariablement fondées sur des absolus d'équivalence quantitative (corrigées d'on ne sait quelles variations par paires de langues) entre constituants du texte ou document premier et constituants du texte ou document second.

L'erreur par distorsion absolue (non-respect des algorithmes de la grammaire culturelle-linguistique) ou relative (non-respect du projet défini par rapport à l'unité stipulée) résulte toujours d'un non-choix ou d'un mauvais choix affectant le cadre analytique prospectif du segment en cours de transfert (projet + organisation générale du trajet) puis le générateur du trajet (objet logique dominant dans l'unité concernée). Il resterait à déterminer quels sont les facteurs de risque (tels que la méconnaissance du domaine de référence) et les facteurs aggravants (tels que la carence de recherche documentaire). Ceci deviendra possible une fois établis les caractères qui s'ajoutent au type (relatif transfert / absolu - rédaction), à la cause (absence de choix / choix défectueux), et à l'origine (cadres, destinations, objets, thèmes, etc.) pour fournir les premiers éléments d'analyse qui serviront plus tard de supports aux indices de pondération.

Ces caractères complémentaires sont la nature et le champ immédiat. La nature de l'erreur (qui décrit souvent déjà la nature de la faute) peut être :

l'omission injustifiée

l'inversion ou la rupture injustifiée

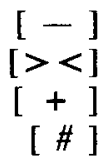

l'ajout injustifié

l'écart injustifié

L'erreur peut, quelle que soit sa nature, avoir pour champ immédiat une unité lexicale/terminologique ou une unité syntaxique.

Les séries de paramètres mobilisés jusqu'à ce point pour tenter de définir les caractères de l'erreur: type (relatif/absolu); origine (cadres/objet/etc.) ; cause (non-choix/choix défectueux) ; nature (omission, ajout, écart, rupture injustifiés) et fondés sur la stipulation de l'unité de référence ultime générant le projet de traduction débouchent sur des combinaisons décrivant chaque erreur possible. Au-delà de ce point, l'erreur devient faute et tout caractère additionnel relève du système de pondération retenu. Lorsque tous les caractères (caractères d'erreur et caractères de faute) ont été définis, on aboutit à la multiplication de séries de paramètres dont les combinaisons permettent de spécifier des centaines de cas d'erreurs correspondant chacun à une 
combinaison donnée d'origine, de type, de champ immédiat, de nature, et d'incidence ou impact direct sur le segment concerné et indirect par propagation d'un éventuel cône de distorsion dans les segments contigus puis distants.

\section{Modes et systèmes d'évaluation}

Une fois les erreurs recensées et caractérisées, il convient habituellement d'évaluer l'impact ou l'effet de la faute résultante. L'observation des résultats d'évaluations parallèles d'une même traduction par plusieurs personnes est, à cet égard, riche d'enseignements. On note que les «correcteurs" s'accordent à «voir les mêmes fautes " aux mêmes endroits, à leur attribuer une même désignation dans la série des catégories universelles du contresens, faux sens, etc., et à fixer une même ligne de démarcation séparant la traduction acceptable (le bon grain) de la traduction non acceptable (l'ivraie). On note surtout qu'ils ne s'accordent presque jamais sur la valeur de pénalité réelle, pondérée, affectant telle ou telle faute et que, en-deçà d'une convergence des jugements de valeurs globaux, la divergence est la règle point par point.

Si l'évaluation globale, intuitive-empirique, suscite les plus forts degrés de convergence, il serait (peut-être) plus sage et (certainement) plus facile de ne prévoir d'évaluation spécifique que pour les cas particuliers... à condition de savoir ce que sont ces cas particuliers dont la nature doit, en toute logique, déterminer la structure des systèmes d'évaluation. Reste aussi, une fois répertoriés les fonctions et objectifs qui lui seront dévolus, à définir l'instrument d'évaluation.

$\mathrm{Au}$ fond, les systèmes d'évaluation des traductions doivent, individuellement ou conjointement, permettre d'évaluer une ou plusieurs traductions dans l'absolu mais aussi d'évaluer les unes par rapport aux autres, plusieurs traductions d'un même document ou plusieurs traductions correspondant chacune à un document spécifique.

L'évaluation appelle ainsi une échelle absolue et une échelle relative utilisées à des fins fort diverses que l'on peut classer en deux catégories: la notation et le contrôle de qualité. Ces deux catégories font l'objet d'applications dans des situations telles la recherche de corrélations entre erreurs et fautes, la définition de stratégies de prévention d'erreurs, la notation "académique », le recrutement ou la promotion de traducteurs, etc. La liste, qui ne recense que quelques cas, suffit néanmoins à suggérer un clivage net entre ce que l'on pourrait appeler les évaluations «à blanc» (sans effet positif ou négatif direct sur le traducteur) et les évaluations "chargées " (avec effet positif ou négatif direct sur le traducteur) qui suscitent souvent la contestation.

La contestation éventuelle des résultats d'une évaluation « lourde de conséquences » incite à préciser très clairement les critères de calcul 
des indices de pondération ou, au contraire, à justifier l'absence de pondération dans une série chronologique d'activités enchaînant :

1. le recensement d'éventuelles erreurs ;

2. le paramétrage des erreurs ;

3. un calcul de pondération ;

4. une notation ou un classement ;

5. la décision résultant de l'évaluation.

Il y a convergence dans le recensement des erreurs dès l'instant où les orientations générales du projet de traduction sont connues. Il y a aussi convergence dans le paramétrage des erreurs quelles que soient les séries utilisées. Le classement ou la notation sont quasi automatiques et la décision résultant de l'évaluation ne vaut que ce que vaut le système utilisé. Seul le calcul des indices de pondération pose problème.

Même si, techniquement, l'évaluation repose sur le calcul de l'incidence des erreurs par application de coefficients aux paramètres d'«erreur-faute », elle s'articule en deux moments qui sont, dans cet ordre, la définition de la base de calcul et l'affectation de coefficients. Les combinaisons entre base de calcul et coefficients déterminent des modes (relativement empiriques) et des systèmes (raisonnés) d'évaluation au nombre de six - trois modes et trois systèmes.

\section{L'intuition et l'habitude}

Le premier mode d'évaluation est celui de l'appréciation subjectiveintuitive globale au terme d'une lecture visant simplement à déclarer la traduction recevable ou non recevable. Il ne nécessite ni recensement, ni caractérisation, ni pondération des erreurs. Il débouche sur une notation ou un classement selon une échelle empirique «interne » à l'auteur de l'évaluation. Il est fort répandu et se cache bien souvent sous des appareils faussement objectifs.

\section{L'indice de révision}

Le second mode d'évaluation des traductions repose sur des critères semi-objectifs de temps de révision. Il appelle la mesure du temps nécessaire à un réviseur donné pour produire une traduction de bonne qualité courante à partir de celle que lui remet le traducteur. Chaque erreur se caractérise par son « indice matériel de révision » mesuré $a$ posteriori.

Si ce second mode d'évaluation pose le double problème de la compétence du réviseur (dont dépend le temps de révision) et de la constance des objectifs (qui peuvent varier selon ses critères définissant la bonne qualité courante), il se justifie pleinement dans tout contexte de traduction suscitant une collaboration " traducteur-réviseur ». Il s'appuie surtout sur la réalité de la pratique qui pousse à réduire les délais 
de production tout en respectant les impératifs de qualité. Appliqué de manière systématique, il débouche sur une modification des procédures de traduction et de pilotage des sous-traitances. Il met clairement en lumière les corrélations entre types de carences dans la démarche du traducteur et facteurs de "perte de temps » imposée au réviseur ou au pilote pour corriger les carences du traducteur (telle carence dans, disons, la recherche documentaire, est pénalisée au prorata du temps que le réviseur consacre effectivement à la recherche que le traducteur n'a pas, ou pas correctement, effectuée). Sous réserve d'adaptation du mode de calcul de l'indice de révision aux conditions toujours spécifiques de chaque institution, bureau, société, groupe, etc., pareil système devrait se généraliser en contexte professionnel et en contexte pédagogique dans la mesure où il ne fait appel à aucun critère extérieur à l'activité de traduction elle-même.

\section{Les conséquences de l'erreur}

Le troisième mode d'évaluation repose sur la notion d'impact des erreurs. La logique est, cette fois, externe à l'activité de traduction proprement dite: les coefficients de pondération affectés aux fautes résultant des diverses erreurs s'établissent selon la gravité de leurs conséquences au niveau des exploitations du texte «traduit». Il s'agit en fait d'un mode d'évaluation appelant sanction extrême des erreurs (quels que soient leur nature et leur nombre dans une traduction donnée et quelle que soit la longueur de cette traduction) que l'on peut dire «fatales» parce qu'elles ont entraîné un refus de la traduction par le donneur d'ouvrage, la perte d'un client par le bureau de traduction, un préjudice matériel, moral ou financier pour le donneur d'ouvrage ou l'employeur, un incident sérieux, un accident, ou tout autre désastre. $^{2}$

On pourrait allonger la liste et établir un lien avec le mode précédent en considérant que l'indice de révision devient également, lorsqu'il est élevé, le signe d'un "manque à gagner" relatif pour le donneur d'ouvrage, le réviseur, le bureau de traduction, l'employeur. Ceci se justifie dans la mesure où le mode d'évaluation considéré ici traduit l'importance de la sanction externe (effective ou envisageable) venue du client ou du donneur d'ouvrage et motivée par des considérations économiques. Accessoirement, ce mode d'évaluation minimise l'impact de toute erreur qui ne nuirait pas à l'exploitation de la traduction ou qui serait automatiquement corrigée par tout utilisateur potentiel.

2. Le mode d'évaluation envisagé ici renvoie à un problème extrêmement complexe qui est celui de la responsabilité du traducteur et du traducteur sous-traitant qui demeure indissociable de la notion d'erreur. 
Ce mode d'évaluation est également envisageable en contexte pédagogique dans la mesure où il permet de faire toucher du doigt les enjeux économiques de la recherche de qualité de traduction. En pareil contexte, on ajoutera aux paramètres économiques directs les paramètres plus flous mais non négligeables de "réputation » liés aux erreurs susceptibles de déconsidérer le traducteur aux yeux du donneur d'ouvrage.

En tout état de cause, ce mode d'évaluation a le mérite de la netteté : il réduit l'effet d'amplification des fautes mineures mais irritantes pour l'évaluateur mais condamne sans appel, comme devrait pouvoir le faire tout mode ou système d'évaluation, toute traduction comportant une erreur lourde de conséquences et non automatiquement rectifiable par tout utilisateur du document traduit. Il permet de «traiter" la faute unique mais gravissime dans une traduction de grande longueur. Il permet de trancher dans les débats portant sur le poids des erreurs. Il rend inutile la mise au point de techniques d'échantillonnage complexes.

Dans la progression des modalités d'évaluation-pondération, on passe ensuite à ce que l'on peut considérer, par opposition aux modes, comme des "systèmes" appelant des calculs relativement complexes.

\section{Du non-sens à l'évanescence}

Le premier système de pondération qui vient à l'esprit est le système standard de la série non-sens, contresens, faux sens, etc., dans lequel les dénominations traduisent déjà une préhiérarchisation des pénalités. D'usage courant, ce système affecte des coefficients différentiels aux catégories universelles de fautes. Les « valeurs » relatives du contresens, du faux sens, etc., ou des inclassables que sont les fautes d'orthographe sont loin de faire l'unanimité. En tout état de cause, le système existe, tout le monde sait coller les étiquettes de catégories voulues sur les fautes, et il suffit de fixer un barème (plus ou moins arbitraire) pour que le système devienne partageable; ce qui, compte tenu des fonctions dévolues aux systèmes d'évaluation, n'est pas un mince mérite.

On peut affiner ce premier système en créant une référence universelle (de longueur et nature de l'échantillon) et en multipliant les catégories auxquelles sont affectés des coefficients. On peut ainsi séparer fautes de langue et fautes de traduction puis, dans chaque groupe, multiplier les sous-catégories. Cependant, quels que soient les raffinements proposés, les catégories restent des catégories absolues. Par conséquent, chaque «faute " relevant d'une catégorie est toujours affectée d'un même coefficient de pénalité quelle que soit son incidence réelle dans le texte alors que, pour simplifier, il est des contresens parfaitement bénins et des faux sens gravissimes. 
Ce premier système d'évaluation n'est autre que le système de notation de la version et du thème que l'on a transposé, faute de mieux, à l'évaluation des traductions pédagogiques puis, hélas, à la pratique professionnelle.

\section{L'effet de distorsion (I)}

Un second type de système d'évaluation des traductions propose l'affectation de coefficients aux divers caractères de chaque faute pris dans les séries précédemment répertoriées. La pénalité correspondant à chaque faute est le résultat de la multiplication d'un indice de base par tous les coefficients affectés aux paramètres caractérisant l'erreur. Pour simplifier, nous dirons que pareil système multiplie une quantité initiale, intrinsèque, correspondant à la nature de la faute par un premier coefficient correspondant à son champ immédiat (introduisant des différences de portée), puis un second correspondant au rôle de l'élément concerné dans le segment affecté par l'erreur, puis un troisième correspondant $\mathrm{au}(\mathrm{x})$ rangs du ou des segments affectés par l'erreur dans le texte. On différencie ainsi, selon le nombre de séries paramétriques et selon l'indice affecté à chaque paramètre de chaque série, des dizaines ou centaines de valeurs de pénalité tentant de rendre compte de l'effet de distorsion.

Ce type de système est particulièrement adapté aux contextes pédagogiques dans la mesure où il explique, depuis son origine jusqu'à son impact spécifique sur la traduction, le mécanisme de l'erreur qu'il décompose. Il permet ainsi de déterminer pourquoi l'erreur se produit, sur quoi elle porte, comment elle s'analyse, et quels sont ses effets négatifs sur la traduction. Il permet aussi de faire varier les coefficients de manière à inciter les sujets à mettre en cuvre au plus vite les stratégies les plus efficaces. Ainsi, pour que chaque traducteur apporte une attention particulière aux mécanismes de recomposition d'un texte, il suffit d'attribuer aux générateurs de segments textuels un coefficient tel que toute erreur affectant ces derniers se convertisse en erreur « fatale».

\section{L'effet de distorsion (II)}

Un troisième type de système d'évaluation repose sur le traitement direct des composantes significatives du texte. Il prend appui sur les inévitables catégories ci-après :

$\begin{array}{lc}\text { absence injustifiée } & {[-]} \\ \text { inversion/rupture injustifiée } & {[><]} \\ \text { présence injustifiée } & {[+]} \\ \text { écart injustifié } & {[\#]}\end{array}$


Les erreurs sont ensuite pondérées en fonction du statut des segments fonctionnels sur lesquels elles se répercutent. On peut distinguer ainsi cinq catégories de segments :

- l'objet logique (ce dont on parle);

- les circonstants de l'objet (ses caractères);

- le thème portant sur l'objet (le commentaire);

- les circonstants du thème (précisions diverses) ;

- les circonstants de l'intégration au texte. ${ }^{3}$

Il suffit d'affecter un indice de pénalité à chaque type d'erreur et de multiplier cet indice par le coefficient raisonné correspondant au statut du ou des segments affectés pour obtenir une valeur de pénalité pour chaque faute.

Pareil système permet de fonder l'évaluation sur des unités simples déterminées par leurs contributions effectives à la forme et au contenu du message et repérées par l'auteur de l'évaluation par induction logique. Il permet de déclasser les erreurs intervenant dans des segments sémantiquement «vides » auxquels aucun effet de multiplication ne s'applique. Il ne nécessite aucune procédure de sélection d'échantillons significatifs. Il démonte le mécanisme de l'incidence de la faute et met en relief les caractères et les effets de la structure profonde, organique, du texte ou document.

Ce type de système convient indifféremment au contexte pédagogique et au contexte de la pratique professionnelle. Il peut surtout, comme le précédent, se transformer en système d'évaluation positive.

\section{L'évaluation «positive »}

Les divers modes et systèmes ci-dessus trouvent, ainsi que nous l'avons signalé, des champs d'application spécialisés. En fait, leur validité serait on ne peut plus nettement confirmée s'ils se transformaient en systèmes d'évaluation positive.

Fondamentalement, tous les modes et systèmes d'évaluation cidessus aboutissent à un total de pénalités et à une «juste rétribution» des fautes éventuellement corrigée d'un «bonus" pour "trouvailles" ou « traductions heureuses ». Le passage à l'évaluation positive mesurant la part de réussite et non la part d'échec exige la mise au point d'un

3. Soit l'exemple schématique ci-dessous :

"Par ailleurs, la traduction directe présente, dans la majorité des cas connus, de fortes ressemblances avec l'interprétation."

Les catégories fonctionnelles "logiques» en sont :

objet $=$ la traduction directe

thème $=$ présente de fortes ressemblances avec l'interprétation

circonstant du thème = dans la majorité des cas connus

circonstants de thématisation $=$ par ailleurs

et il n'y a pas de circonstant d'objet. 
indice de référence que nous dirons indice de qualité-quantité de traduction requise. Si l'indice de traduction requise est égal à $\mathrm{T}$ et le total des pénalités est égal à $\mathrm{P}$, la quantité de «réussite » est égale à $\mathrm{T}-\mathrm{P}$ et le taux de réussite $\mathrm{R}$ est égal à

$$
\frac{\mathrm{T}-\mathrm{P}}{\mathrm{T}}
$$

Deux voies permettent de déterminer l'indice $\mathrm{T}$ de référence. La première conduit à rendre positif le système visant à évaluer l'incidence des fautes; la seconde conduit à rendre positive l'évaluation pondérée selon les segments fonctionnels des segments touchés par l'erreur.

Dans le premier cas, il s'agit de déterminer les caractères significatifs des textes et des traductions et de leur attribuer les coefficients requis. À titre indicatif, la densité terminologique, la complexité des phrases, la présence et la fréquence des séparateurs sémantiques, la présence et la fréquence des facteurs de cohésion, le degré de stéréotypie, déterminent (en conjonction avec bien d'autres facteurs) la difficulté de la traduction. Les calculs sont, à n'en pas douter, fort complexes mais il faudra sans doute un jour y recourir si l'on veut que la pondération soit «juste». En effet, les coefficients attribués aux caractères du texte à traduire (indice de difficulté de traduction) sont automatiquement corrélés aux coefficients attribués à certains caractères des erreurs-fautes (disons, pour simplifier, qu'une faute "grave» verra sa gravité atténuée si elle intervient dans un contexte de grande difficulté de traduction). Il faudra assurément partir du laboratoire de recherche pour aller, progressivement, vers des applications professionnelles allégées. Mais il sera difficile de construire un système viable sans s'inspirer du principe.

Dans le second cas, l'indice de référence s'obtient en additionnant les «points» correspondant à l'ensemble des unités thématiques que comporte le texte à traduire. Le calcul est, cette fois, d'une très grande simplicité puisque l'on peut arbitrairement décider que chaque unité a une valeur de - disons - 10 points à multiplier par le nombre d'unités présentes, pour obtenir l'indice de quantité-qualité de traduction requise. Il suffit ensuite de déterminer les "parts" de contributions respectives des composants de l'unité thématique (segments fonctionnels précédemment pris en compte) pour obtenir les coefficients affectés, dans le calcul de pénalités, aux valeurs initiales des erreurs intervenant dans tel ou tel type de segment. Si l'on considère, par exemple, que l'« objet» vaut 3 et le «thème » 5 , une même faute aura le coefficient 5 si elle porte sur le thème et 3 si elle porte sur l'objet ${ }^{4}$. Le total des 
pénalités est, ici encore, rapporté au total des «points » initiaux correspondant à l'indice de qualité-quantité de traduction requise pour donner un taux de réussite.

La seconde voie de l'évaluation positive est infiniment plus simple que la première. Moins précise, elle conserve néanmoins toutes les vertus que l'on peut reconnaître aux systèmes pondérés.

Le problème crucial que posent la sélection des paramètres de calcul de l'indice de référence et le calcul de leurs coefficients respectifs ne sera pas aisément résolu. Les deux références absolues utilisables dans la mesure des effets des erreurs sont la carence ou perte de compréhension du document ou texte second, d'une part, et l'indice de révision, d'autre part. La mise au point d'un système à la fois maniable et juste exigera de longues recherches mais c'est seulement à ce prix que l'on pourra, lorsque ceci devient absolument nécessaire, échapper à l'intuition, à l'introspection, à l'empirisme, et, en tout état de cause, poser les fondements vrais d'une science et d'une didactique de la traduction.

\section{Applications ; constats ; perspectives}

En attendant que les systèmes parfaits, ultra-didactiques, simples d'utilisation, rigoureux, incontestables, voient le jour, il est bon de rappeler que, même en cas d'utilisation d'instruments relativement rudimentaires, les évaluations des traductions doivent expliquer l'erreur, fixer son incidence, permettre de la prévenir et/ou d'empêcher la propagation du cône de distorsion, et fournir les moyens du contrôle de qualité. $\mathrm{Si}$ ces objectifs et fonctions sont diversement interprétés selon les conditions particulières d'exploitation de modes et systèmes d'évaluation, trois constantes doivent prévaloir.

\section{L'évaluation des traducteurs}

L'évaluation des traducteurs ne peut se réduire à une mesure brute de la qualité (ou du manque de qualités) de leurs traductions. Par ailleurs, aucune situation d'évaluation ne devrait réserver l'exclusivité absolue à tel mode ou système. Enfin, les évaluations devraient s'organiser par niveaux ou paliers de qualité.

Sauf en situation d'erreur fatale, le plus grand danger peut venir, dans la mise en cuvre de systèmes d'évaluation, de procédures qui s'arrêtent à la mesure brute d'un indice de pénalité ou de réussite de traduction constituant la seule motivation d'une décision concernant le traducteur. Il faut que l'évaluation débouche sur une indication du potentiel du traducteur, de ses points forts, et de ses carences, afin que puissent être créées les conditions de l'amélioration de ses performances. 
Pour être significatives, les évaluations doivent être multiples et diverses. Il serait ainsi souhaitable que toute traduction soit soumise à des modes d'évaluation reposant sur l'erreur «fatale " et à un ou plusieurs systèmes élaborés prenant en compte des modalités de pondération fine. Il serait surtout éminemment souhaitable que les traducteurs en formation soient aussi (mais surtout pas exclusivement) soumis à des évaluations correspondant aux schémas les plus brutaux des contextes de la pratique professionnelle et, notamment, à des évaluations arbitraires, subjectives et n'appelant pas justification des pénalités. Et pourquoi ne pas exploiter, en contexte de formation, un régime d'évaluation dans lequel les rétributions sonnantes et trébuchantes seraient diminuées des éventuelles rémunérations de quiconque reprendrait tout ou partie de telle ou telle activité mal conduite ? L'expérience est fort probante.

\section{Les seuils de qualité}

Mais le plus important, dans l'application de modes ou systèmes d'évaluation, reste la définition de niveaux ou paliers de qualité enchaînant :

a) la traduction non révisable - refusée (à reprendre);

b) la traduction acceptée pour révision (révisable);

c) la traduction livrée (livrable).

Les paliers, ainsi que leurs caractères définitoires, doivent être stipulés et figurer, le cas échéant, au cahier des charges, accompagnés des modalités de test (nature et extraction des échantillons, principe de recensement d'erreurs, principes de pondération de pénalités, seuils fixés, etc.). La stipulation peut prendre la forme d'une liste des traitements obligatoires pour qu'une traduction soit, par exemple, déclarée révisable. Ainsi, en règle générale, une traduction sera déclarée révisable si elle a fait l'objet d'un pointage permettant de vérifier qu'elle ne comporte pas autre chose qu'une proportion «irréductible» d'omissions, de fautes de frappe, de fautes d'orthographe, d'accord, de syntaxe, de ponctuation, etc., qu'elle respecte, dans sa forme et sa présentation (alinéas, pagination, enrichissements de caractères, formats, etc.) les directives du donneur d'ouvrage ou les données du cahier des charges, qu'elle n'inclut pas de propositions ou phrases tronquées, incomplètes, incompréhensibles, ou avec éléments parasites, et, enfin, qu'elle respecte en tout point la cohérence terminologique. Indépendamment de ces exigences absolues, une traduction peut être renvoyée au traducteur si elle comporte un taux excessif (à préciser) d'erreurs de transfert (de types à préciser). Le seuil à partir duquel un texte peut être confié au réviseur appelle détermination d'un indice spécifique et se justifie par la nécessité de « rentabiliser » l'intervention du réviseur en s'assurant qu'il lui reste simplement à contrôler la 
congruence technique-linguistique-rhétorique, à la fois dans l'absolu et par référence à un document ou texte premier.

Les seuils de qualité révisable peuvent correspondre à des niveaux d'exigence fort divers mais l'objectif est, invariablement, de réduire la part d'intervention non spécialisée du réviseur. L'exception étant acceptée, la vérification de conformité aux critères du "révisable " peut s'effectuer par échantillonnage dans la mesure où ce qui importe n'est pas tant la présence de l'erreur que sa fréquence et que toute erreur fréquente est détectable dans des échantillons sélectifs.

Les seuils de qualité livrable se situent, bien entendu, au-delà des seuils de qualité révisable. En principe, une traduction livrable devrait présenter une qualité "zéro défaut" mais, ceci constituant l'utopie, on peut estimer qu'une traduction est livrable :

a) du point de vue du donneur d'ouvrage : dès l'instant où elle ne comporte aucune erreur susceptible de nuire à l'exploitation de la traduction par l'un au moins de ses utilisateurs possibles. Elle ne doit donc comporter aucune erreur risquant de provoquer une rupture de la compréhension ou de l'interprétation du texte ou une distorsion que l'utilisateur ne pourrait rectifier d'emblée.

b) du point de vue du traducteur: dès l'instant où elle ne comporte ni erreur du type ci-dessus, ni erreur susceptible de déconsidérer la traduction (et peut-être le traducteur) aux yeux de quelque utilisateur que ce soit. Les deux types d'erreurs se confondent souvent mais il est bon de savoir que le second type est au moins aussi important que le premier dans la mesure où la faute d'orthographe ou d'expression est, pour beaucoup de techniciens, l'indice d'une incompétence générale.

Quel que soit le point de vue retenu, la définition des seuils de qualité livrable ne peut en aucun cas s'accommoder de l'échantillonnage puisque l'erreur «fatale» peut, en exemplaire unique, se dissimuler en n'importe quel point d'une traduction comportant des centaines de pages.

\section{Les paliers pédagogiques}

Il faudrait sans doute, pour aller jusqu'au bout d'une logique évoquée antérieurement, suggérer au pédagogue de définir ses propres paliers ou niveaux de qualité "révisable» et « livrable " en fixant des objectifs ultimes aussi proches que possible des critères professionnels. Il faudrait aussi, mais l'entreprise est infiniment plus complexe, fixer des paliers correspondant aux divers niveaux de compétence balisant l'évolution attestée par la représentation d'un profil évolutif reposant sur une analyse des types d'erreurs commises. Ceci devrait, par contrecoup, permettre de poser des objectifs absolus puis de construire et évaluer les stratégies adéquates visant à l'élimination des types d'erreurs (et 
des comportements qui les induisent) caractérisant un niveau donné avant de passer au niveau suivant. On pourrait ainsi, par le biais d'une redéfinition des critères et modalités d'évaluation, réorganiser aussi la pédagogie.

En effet, si les systèmes d'évaluation des traductions doivent servir en priorité au contrôle de qualité en contexte professionnel et à l'analyse des processus de la traduction en contexte de recherche, il faut savoir que toute erreur résulte toujours d'un comportement non maîtrisé. La qualité en traduction s'obtient donc par la mise en œuvre d'un schéma simplissime aux termes duquel le traducteur doit, pour chacun des niveaux d'emboîtement précédemment considérés et en commençant par le niveau générique:

a) définir ses objectifs ;

b) percevoir et analyser les contraintes;

c) construire des stratégies efficaces ;

ou encore :

a) délimiter les cadres;

b) définir le projet de (segment de) texte ;

c) définir les éventuelles rubriques ;

d) élaborer les éléments du trajet ;

e) sélectionner les générateurs (objets);

f) construire des thèmes - rédiger ;

g) intégrer tous les circonstants voulus.

Il appartient alors à l'enseignant (et au praticien) de déterminer et mettre en cuvre tous les moyens nécessaires pour :

i) éliminer tout risque d'erreur

- en définissant, proposant, ou appliquant toute stratégie nécessaire de compréhension, formulation et vérification d'inférences, construction d'index documentaire, recherche documentaire, etc. ;

ii) éliminer tout facteur aggravant

- en analysant soigneusement les comportements des sujets ;

iii) limiter l'incidence de toute erreur éventuelle

- en définissant et respectant les conditions garantissant qu'il ne puisse y avoir, par l'entremise du traducteur ou du réviseur-correcteur (induit en erreur par une fausse certitude du traducteur), formation et propagation d'un cône de distorsion.

Pour démonter les enchaînements de type « facteur de risque + facteurs aggravant le risque + origine de l'erreur éventuelle + cause + nature + impact ou incidence + modes de traitement par évaluation ", il faut remonter très loin en amont de l'erreur effective constatée 
et analyser les comportements induisant l'erreur (et non pas les "pièges » de la traduction), les comportements aggravant les facteurs de risque (et dont certains sont pour le moins étonnants) ${ }^{5}$, et les comportements de "gestion" des cônes de distorsion. À partir de ces résultats se construiront les bases d'une progression complète, raisonnée, des apprentissages nécessaires. La démarche n'est envisageable que si l'on dispose d'un système de décomposition des mécanismes générateurs d'erreurs et de leurs effets permettant de construire et suivre l'évolution des comportements en fonction de variations de telle ou telle condition négative (facteur de risque ou facteur aggravant) ou positive (effet de telle ou telle stratégie). On dépassera ainsi le stade de l'empirisme pour déterminer les éventuelles corrélations positives ou négatives entre la cause (comportement) et les effets (qualité de traduction).

Parmi les défis qui seront alors posés aux formateurs, on citera la recherche d'une solution au conflit entre la nécessité de donner à la traduction tous les caractères organiques requis et la mise en cuvre de mécanismes visant à prévenir ou contrôler la formation et la propagation d'éventuels cônes de distorsion générés par l'erreur. Conduite à l'aide d'un système d'évaluation relativement fin, l'analyse des comportements et de leurs effets confirme en effet que l'omniprésence du risque d'erreur induit des comportements qui sont en totale contradiction avec les impératifs de cohésion et cohérence textuelle ou avec les impératifs de la révision-correction. Le traducteur tend à fragmenter ses unités de transfert et à clore (verrouiller) chacune d'entre elles pour limiter le champ d'extension de toute erreur éventuelle : la crainte des répercussions de l'erreur empêche l'exploitation naturelle des facteurs de cohérence et de cohésion. Il faudra que la pédagogie de la traduction prenne en compte l'erreur née de la crainte de l'erreur, confinée à la plus petite unité artificiellement verrouillable, et difficilement détectée par le réviseur dont les préoccupations vont nécessairement vers l' «injection " de facteurs de cohésion et de cohérence textuelle dans un enchaînement déconstruit. Il faut, en d'autres termes, commencer à s'interroger sur les effets négatifs de la crainte de l'erreur.

Le « réflexe» de verrouillage des unités de traduction créant une « enceinte de confinement» de toute erreur éventuelle témoigne d'une perception instinctive mais forte du chaînage naturel des erreurs déterminé par la structure d'enchâssement des constituants d'un texte ou document. L'enceinte de confinement se crée par verrouillage de toute

5. Sait-on, par exemple, qu'il existe chez les apprentis-traducteurs une très forte résistance à l'exploitation des résultats de la recherche documentaire et que ce que l'on prend parfois pour absence de documentation est en fait refus de, ou incapacité à, intégrer les résultats d'une activité effectivement et convenablement conduite? 
unité de haut niveau ou par multiplication des unités de bas niveau juxtaposées et désolidarisées les unes des autres. On ne s'étonnera guère que l'un ou l'autre moyen engendre un effet de déconstruction textuelle. Il faut, pour y remédier, analyser de manière rigoureuse les conduites et les performances de l'apprenti-traducteur.

L'analyse des performances des traducteurs et apprentis-traducteurs à l'aide d'un système de décomposition des erreurs et de leurs mécanismes fait apparaître un certain nombre de données d'un intérêt capital :

* il existe, dans l'évolution des performances du traducteur, des paliers homogènes, très nettement caractérisés, et que l'on peut recréer en plaçant, par exemple, un traducteur confirmé en situation de carence de savoirs ;

* les paliers ou niveaux de performance s'enchaînent selon un principe de conditions nécessaires (mais non suffisantes) de passage aux paliers supérieurs ;

* les paliers ou niveaux de performance correspondent à des types de produits répondant à la définition d'autant de types naturels de tâches de traduction;

* les passages de palier en palier ne sont pas linéaires mais sont prévisibles et peuvent être accélérés ;

* les paliers ou niveaux sont directement corrélés à la taille des unités prises en compte ;

* certaines erreurs de traduction sont des erreurs inévitables provoquées par un dépassement forcé des paliers de compétence.

La définition d'un cursus de formation éliminant les comportements d'erreur devrait s'organiser selon les paliers successifs et accélérer les passages d'un niveau à l'autre en réduisant à la fois les risques, les facteurs aggravants, et les impacts. Il suffit de traiter les apprentissages selon un système de stratifications ou couches horizontales et non par accumulation de points ou d'objectifs juxtaposables. Il suffit d'enchaîner la traduction signalétique, la traduction sélective, les diverses variantes de la traduction synoptique, la traduction diagrammatique, les traductions banalisées, les traductions post-synthétiques, et les traductions absolues. ${ }^{6}$

Il faudra alors porter la plus grande attention à un phénomène apparemment négligé : la régression positive marquée par des erreurs mais signalant le passage imminent au palier supérieur. Il en va ainsi de la phase de déstabilisation syntaxique-collocative précédant la maî-

6. Voir, du même auteur, "Autrement dire - pour une redéfinition des stratégies de formation des traducteurs" (à paraître dans META). 
trise du paragraphe logique. Le phénomène intéresse en fait toute personne directement ou indirectement concernée par l'erreur en traduction. Il est probable que la définition d'instruments rigoureux de décomposition, analyse et pondération des erreurs débouchera très rapidement sur la mise en évidence d'autres phénomènes hautement significatifs mais masqués, jusqu'ici, par le caractère rudimentaire des moyens d'analyse de l'erreur et de mesure de la faute.

\section{Conclusion}

Quoi de plus urgent, au fond, que de définir l'erreur et de trouver les moyens de mesurer ses effets? Mais aussi, quoi de plus difficile? Si la liste des postulats sur lesquels devrait reposer la gestion de la notion d'erreur se construit aisément, on ne peut guère que dégager des caractères de l'erreur et poser les principes selon lesquels on peut décider qu'il y a erreur. La notion d'erreur se lie ainsi indissolublement à la notion de projet de traduction arrêté à l'unité considérée comme fonctionnellement insécable. L'erreur absolue (communication) et l'erreur relative (transfert) renvoient, en pratique, à des décompositions par séries de paramètres « utiles ». Ces dernières ayant été répertoriées, il faut tenter de résoudre le redoutable problème de la "mesure" de l'effet ou impact des fautes. Les réponses brutes possibles conduisent à caractériser divers modes et systèmes répondant à des logiques diverses, à des exigences diverses, à des situations privilégiées. Nous ne pouvons, en matière de choix et d'application de mode ou système d'évaluation, que plaider en faveur des évaluations positives dont les orientations confirment qu'il faudra constituer, analyser, et pondérer de nombreuses séries de paramètres pour répondre aux diversités des situations (enseignement, recherche, pratique) dans lesquelles s'imposent la définition de l'erreur, la mesure de la faute, et la compréhension des effets de l'une et de l'autre sur les comportements du traducteur. Nous pensons que l'analyse de la notion d'erreur doit conduire à une redéfinition des orientations de recherche, des modalités d'évaluation professionnelle, et des pratiques pédagogiques. Nous n'avons, dans ces quelques pages, fait qu'effleurer un domaine infini.

Université de Rennes 2 